\title{
Toward a definition of awareness
}

\author{
PHILIP M. MERIKLE \\ University of Waterloo, Waterloo, Ontario, Canada
}

\begin{abstract}
Two different current definitions of awareness are contrasted. One definition, used in recent masked-prime studies, is objective and equates awareness with the ability to make forced-choice decisions above a chance level of performance. The second definition, proposed by Henley (1984), is subjective and simply equates awareness with self-reports indicating that an observer "consciously sees" a stimulus. It is concluded that a better objective measure of awareness is needed to distinguish the subjective states of "seeing" and "not seeing" a stimulus.
\end{abstract}

Henley (1984) recently commented on a paper I wrote (Merikle, 1982) criticizing the methodology employed in studies that appear to indicate that masked, visual priming stimuli are effective even when observers can neither identify (McCauley, Parmelee, Sperber, \& Carr, 1980) nor detect (Balota, 1983; Fowler, Wolford, Slade, \& Tassinary, 1981; Marcel, $1983)$ the primes. In these studies, awareness was defined as the ability to make better than chancelevel, forced-choice decisions concerning either the identity or the presence of the primes, and, conversely, it was assumed that observers were unaware of the primes when these decisions were at a chance level of performance. The surprising result was that the effectiveness of the priming stimuli did not appear to depend on the level of awareness, in that equivalent priming effects were observed when the primes were presented above or below the presumed awareness threshold. If awareness thresholds, as indicated by chance-level, forced-choice performance, had been established by adequate psychophysical procedures, then these results would provide strong support for the perception-without-awareness hypothesis. However, the procedures used in these studies to establish awareness thresholds were inadequate (Merikle, 1982), and thus the reported data do not provide convincing evidence that perceptual processing occurs in the absence of awareness.

Although Henley (1984) agrees that the procedures used in these studies to establish chance-level, forcedchoice performance were inadequate, she argues that the critique was misdirected. According to Henley, what should have been emphasized was that these studies investigating the effects of masked primes are irrelevant to questions concerning the perception-withoutawareness hypothesis because awareness was defined in an inappropriate manner. Henley claims that forcedchoice decisions can be made above a chance level of

Preparation of this article was supported by Grant APA-231 from the Natural Sciences and Engineering Research Council of Canada. The author's mailing address is: Department of Psychology, University of Waterloo, Waterloo, Ontario, Canada N2L 3G1. performance even when observers state that they cannot "consciously see" the stimuli. For this reason, she concludes that it is inappropriate to define awareness in terms of a threshold for discriminated verbal reports and that therefore the critical assumption underlying the recent masked-prime studies is incorrect. Given this disagreement, an explicit contrast between Henley's suggested definition of awareness and the definition adopted in recent masked-prime studies should serve to sharpen discussion of the definitional issues involved in this controversial research area.

The main reason that the recent masked-prime studies have had a particularly strong impact is that an objective definition of awareness was used. Awareness was defined in terms of an observer's ability to discriminate among several possible stimulus states, as indicated by verbal reports in forced-choice tasks. Thus, an attempt was made in these studies to correlate an objectively specified stimulus state (e.g., the presence or absence of a stimulus) with an objectively specified response (e.g., the verbal response "present" or "absent"). It was assumed that when the correlation between stimulus states and responses approximated zero, observers were unaware of the stimuli. Conversely, when the correlation was greater than zero, it was assumed that the observers were aware of the stimuli. ${ }^{1}$

This definition of awareness is completely consistent with the position put forward by Eriksen (1960) in his influential paper concerning methodological considerations underlying subception research. A definition based on forced-choice decisions has several distinct advantages: (1) it is objective, (2) it is readily implemented in different laboratories, and (3) it provides a method for assessing perceptual sensitivity independent of response bias. If perceptual processing could be shown to occur when an observer cannot discriminate among the possible alternative stimuli, this would be strong evidence for the perception-without-awareness hypothesis, because it would contradict Eriksen's earlier conclusion that verbal reports are as sensitive an indicator of perceptual processing as any other response that has been studied.

In contrast to the approach adopted in the recent 
masked-prime studies, Henley (1984) suggests that awareness can be measured adequately by simply asking observers whether or not they "consciously see" a stimulus. Thus, if an observer reports no awareness of a stimulus, then, by definition, the observer is unaware of the stimulus. Conversely, if an observer reports that a stimulus is "consciously seen," then, by definition, the observer is aware of the stimulus. This definition of awareness is exactly the same as the one espoused by Dixon (1981) in his recent monograph. The fundamental problem with this definition, as I have discussed elsewhere (Merikle, 1983), is that it transfers the responsibility for operationally defining awareness from the investigator to the observer. Any experimenter who uses this definition is, in effect, asking each observer to provide his or her own definition of awareness.

Although Henley (1984) implies that her suggested definition of awareness is based upon discriminated verbal reports, she applies this label to a situation that is completely different from the one described by Eriksen (1960) and used in recent masked-prime studies. Eriksen referred to a situation in which observers are presented different stimulus conditions, and their ability to provide a different response for each stimulus condition is measured. Henley, on the other hand, applies the label "discriminated verbal report" to a situation in which a single stimulus is presented and any differential responding that may occur is based on the observers' self-reports of their conscious experiences.

The weaknesses in Henley's (1984) definition of awareness are obvious. In addition to being completely circular, her definition is subject to the same criticisms that can be directed at any study of perceptual processes based solely on observers' introspective reports. Awareness thresholds, as measured by self-reports, will vary tremendously depending upon the demand characteristics in a particular experimental situation. For example, an experiment is not needed to demonstrate that observers will have a different awareness threshold when they are paid $\$ 100$ each time they consciously detect a stimulus than when they are penalized $\$ 100$ each time they fail to detect a stimulus. More subtle demand characteristics communicated by an experimenter, as well as an observer's preconceptions concerning an experiment, will also influence observed awareness thresholds, if Henley's suggested definition of awareness is adopted. Thus, defining awareness in terms of introspective selfreports will only make it difficult to replicate particular patterns of results, both within the same laboratory and across different laboratories, and, in general, will lead to endless controversy concerning whether or not perception without awareness actually occurs. In fact, the long-standing controversy concerning this phenomenon, as documented in Dixon's $(1971,1981)$ monographs for 11 different research areas, can probably be attributed to the fact that far too many investigators have defined awareness solely in terms of subjective criteria.
Even though Henley's (1984) suggested definition of awareness is unacceptable, this does not necessarily imply that awareness is best defined as an ability to make forced-choice decisions and that nonawareness is the absence of such an ability. It is entirely possible that Henley is correct in stating that forced-choice decisions, at least under certain conditions, may be made in the absence of awareness. However, even if her supposition concerning forced-choice decisions is correct, the solution to the definitional problem is not to substitute a subjective definition for an objective one. Rather, the solution lies in the development of an objective definition of awareness that clearly distinguishes the subjective state of "not consciously seeing" a stimulus, which many observers experience when they are shown highly degraded visual stimuli, from the more normal subjective state of "consciously seeing" a stimulus (cf. Cheesman \& Merikle, 1983).

\section{REFERENCES}

Balota, D. A. (1983). Automatic semantic activation and episodic memory encoding. Journal of Verbal Learning and Verbal Behavior, 22, 88-104.

Cheesman, J., \& Merikle, P. M. (1983, November). Distinguishing conscious from unconscious processes. Paper presented at the meeting of the Psychonomic Society, San Diego.

Dixon, N. F. (1971). Subliminal perception: The nature of a controversy. London: McGraw-Hill.

Dixon, N. (1981). Preconscious processing. Toronto: Wiley.

Eriksen, C. W. (1960). Discrimination and learning without awareness: A methodological survey and evaluation. Psychological Review, 67, 279-300.

Fowler, C. A., Wolford, G., Slade, R., \& Tassinary, L. (1981). Lexical access with and without awareness. Journal of Experimental Psychology: General, 110, 341-362.

HenLEY, S. H. A. (1984). Unconscious perception re-revisited: A comment on Merikle's (1982) paper. Bulletin of the Psychonomic Society, 22, 121-124.

MarCEL, A. J. (1983). Concious and unconscious perception: Experiments on visual masking and word recognition. Cognitive Psychology, 15, 197-237.

McCauley, C., Parmelee, C. M., Sperber, R. D., \& Carr, T. H. (1980). Early extraction of meaning from pictures and its relation to conscious identification. Journal of Experimental Psychology: Human Perception and Performance, 6, 265-276.

MerikLe, P. M. (1982). Unconscious perception revisited. Perception \& Psychophysics, 31, 298-301.

MERIKLE, P. M. (1983). Subliminal perception reaffirmed. A review of Preconscious Processing by Norman Dixon. Canadian Journal of Psychology, 37, 324-326.

\section{NOTE}

1. The criterion of zero correlation between stimulus states and responses has not been used to define awareness thresholds in all masked-prime studies. Marcel (1983), for example, defined the awareness threshold as $60 \%$ correct in a two-alternative, forced-choice task. This level of performance suggests that the subjects in Marcel's experiments could detect the presence of the masked primes, contrary to the widely held interpretation of these findings.

(Manuscript received for publication May 28, 1984.) 